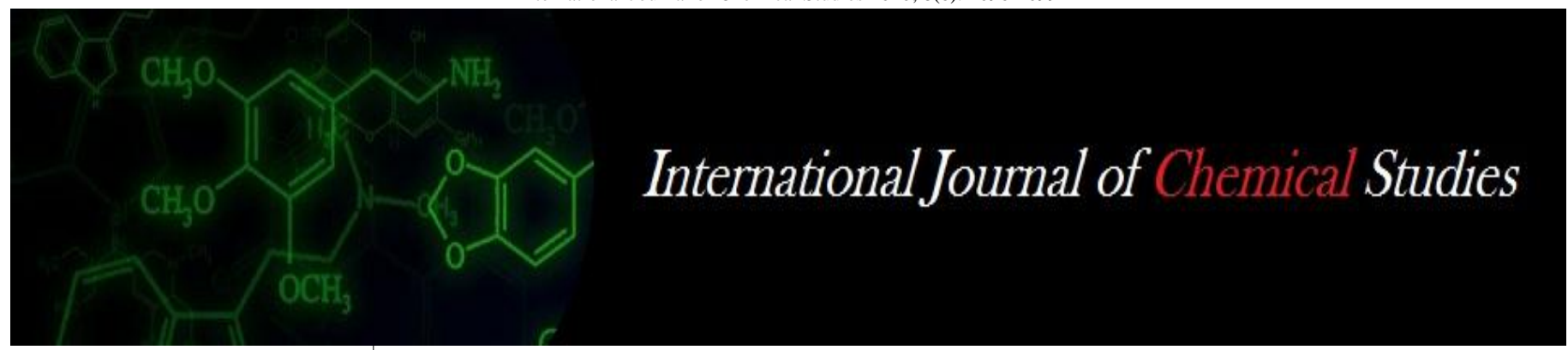

P-ISSN: 2349-8528

E-ISSN: 2321-4902

www.chemijournal.com

IJCS 2020; 8(6): 1096-1099

(C) 2020 IJCS

Received: 22-08-2020

Accepted: 10-10-2020

\section{SE Manjunatha}

Ph. D. Scholar, College of

Agriculture, (UAS Dharwad),

Dharwad, Karnataka, India

\section{Rajegowda}

Senior Scientist and Head, KVK,

Kandali, (UAS-Bangalore),

Hassan, Karnataka, India

\section{Raju}

Professor of Sericulture, College of Agriculture, (UAS-Bangalore), V.C. Farm, Mandya, Karnataka, India

\section{N Kiran Kumar}

Associate Professor, Department of Plant Pathology, College of Agriculture, (UAS-Bangalore), V.C. Farm, Mandya, Karnataka, India

VB Sanath Kumar

Professor, Department of Plant Pathology, College of

Agriculture, (UAS-Bangalore),

V.C. Farm, Mandya, Karnataka, India

\section{KR Ashoka}

Assistant Professor, Department of Soil Science and Agricultural Chemistry, College of Agriculture (UAS-Bangalore), V.C. Farm Mandya, Karnataka, India
Corresponding Author: VB Sanath Kumar

Professor, Department of Plant Pathology, College of Agriculture, (UAS-Bangalore),

V.C. Farm, Mandya, Karnataka, India

\section{Performance variation in growth and sporulation of isolates of Alternaria alternata Fr. Keissler causing blight disease in Mulberry}

\author{
SE Manjunatha, Rajegowda, M Raju, N Kiran Kumar, VB Sanath Kumar \\ and KR Ashoka
}

DOI: https://doi.org/10.22271/chemi.2020.v8.i6p.10907

\begin{abstract}
Mulberry Alternaria blight (Alternaria alternata) is one of the foliar devastating diseases and reduces leaf quality and yield. Studying the cultural characters of the pathogen is the first and most important basic step in identification process. The results of cultural studies revealed that PDA was the best medium for supporting the good radial growth of the isolate Aa-1, Aa-2, Aa-4 and Aa-7 with the maximum radial growth of $90.00 \mathrm{~mm}$ after ten days of inoculation. Whereas, isolate Aa-2 and Aa-6 yielded maximum growth of $90 \mathrm{~mm}$ in Sabouraud's dextrose agar medium and isolate Aa-8 and Aa-9 in oat meal agar medium. Maximum growth $(90 \mathrm{~mm})$ in corn meal agar (Aa-7) and in Richards synthetic agar medium isolate Aa-4, Aa-6 and Aa-10 recorded good growth. Dry weight of mycelium of the different isolates $A$. alternata studies revealed that isolate Aa-3 showed maximum dry mycelial weight of the fungus noticed in Host leaf extract broth $(998.71 \mathrm{mg}$ ) which was followed by Isolate Aa-2 $(997.93 \mathrm{mg})$ and Isolate Aa-5 (997.4mg) in Sabouraud's dextrose broth. Very good sporulation of the Isolate Aa-1 on Potato dextrose broth, Host leaf extracts broth, Oat meal agar broth and Corn meal broth was recorded. The Potato broth also supported good sporulation of three isolates viz: Aa-1, Aa-5 and Aa-9 whereas, by Host leaf extract broth and corn meal broth supported good sporulation of Isolate Aa-4 and Aa-5 respectively.
\end{abstract}

Keywords: Alternaria blight, Alternaria alternate, solid media, liquid media, cultural variability

\section{Introduction}

Mulberry (Morus alba) is one of the commercial perennial plant grown in topical India. The healthy leaves of mulberry are the only food source for silkworm (Bombyx mori L.) and it belongs to the family Moraceae. It is cultivated in both tropical and temperate countries of the world. Though mulberry cultivation is practiced in various climates in India, it is extensively grown in the tropical zone covering Karnataka, Andhra Pradesh and Tamil Nadu states with about 90 percent of area where, most of the sericulture industry is concentrated.

Healthy mulberry leaves are the one of the chief source of morine protein for Silk worm and leaf blight incited by Alternaria alternata adversely affects the protein content and yield of quality leaves. It manifests by the appearance of burning at leaf tip initially and spread towards the middle which later become brownish-black. Infected leaves dries up within a week and ultimately mulberry growth affected, which lead to poor leaf quality and decrease the leaf yield. The disease also affects the seedling by causing seedling blight. The fungus belongs to the Class Deuteromycetes. The spores of A. alternata are five to six horizontal septa with two to three vertical septa and pigmented and they are produced in chains or branching chains. The spores have a distinctive appearance that makes them easy to recognize. They are broadest near the base and taper gradually to a short beak. The disease is common in southern part of India during Kharif season. The disease cause severe defoliation and mortality during winter months (Gunasekhar and Govindaiah, 1990-91) ${ }^{[2]}$.

Since the information on the growth and sporulation of the fungus on different nutritional sources is scanty, present study was undertaken to know the variation among different isolates of Alternaria alternata in mulberry growing areas of Mandya district with respect to growth parameters on various culture media. 


\section{Materials and methods}

The study was conducted to assess the growth variability of $F$. verticillioides isolates on different media during 2017 at Department of Plant Pathology, College of Agriculture, V.C. Farm, Mandya.

\section{Isolation of the different isolates of Alternaria alternata}

Different isolates of Alternaria alternata leaf blight of mulberry samples were collected from ten different locations viz: Malavally (Aa-1), Channapatna (Aa-2), Ramanagara (Aa3), Magadi (Aa-4), Madduru (Aa-5), Mandya (Aa-6), Nagamangala (Aa-7), Krishna raja Pete (Aa-8), Sri ranga Patna (Aa-9) and Mysore (Aa-10) and the isolate name was given accordingly. The isolation was done by standard tissue isolation method. The Pathogen was identified by comparing their morphological characters of vegetative and reproductive structures on potato dextrose agar Petri plates with the standard descriptions described by Leslie and Summerell $(2006)^{[3]}$.

Growth studies of $A$. alternata isolates on different solid and liquid media

The eight solid media viz; Potato dextrose agar, Host Leaf Extract Agar, Sabouraud's dextrose agar, Takahashii's medium, Oat meal agar, Corn meal agar, Richard's synthetic agar and Yeast extract agar were prepared. $5 \mathrm{~mm}$ disc from seven day old culture of each A. alternata isolates were placed in the centre of Petri plates containing test media and were incubated at $28 \pm 1^{\circ} \mathrm{C}$ for eight days and radial growth was measured. Similarly the same eight liquid media were used to test the growth variability of the isolates. A $100 \mathrm{ml}$ of each respective prepared broth contained in $250 \mathrm{ml}$ conical flasks was inoculated with $5 \mathrm{~mm}$ disc of each isolate and incubated at $28 \pm 1^{\circ} \mathrm{C}$ for eight days. Later, the broth was filtered using $12.5 \mathrm{~cm}$ diameter Whatman No 1 filter paper discs. The mycelial mat along with the filter paper was dried at $60^{\circ} \mathrm{C}$ in a hot air oven. Finally, the weight of the harvested mycelia mat and sporulation were recorded. Average of dry mycelial weight of the three treatments was calculated to compare the effect of different liquid broth on mycelia dry weight of fungus.

\section{Sporulation studies of $A$. alternate isolates on different media}

Sporulation was estimated by dissolving the eight days old 5 $\mathrm{mm}$ disc of pathogen culture grown on different media, in 10 $\mathrm{ml}$ of distilled water and shaken well to obtain the spores in solution. The spores were counted with the help of compound microscope under 10x magnification. The results were expressed as very good, good, fair, poor and no sporulation on the basis of the following scale as mentioned in Table 1.

Table 1: Details of expression of sporulation of mulberry blight pathogen

\begin{tabular}{|c|c|c|}
\hline Sporulation & Representation & No of conidia/10x microscopic field \\
\hline Very good & ++++ & $>40$ \\
\hline Good & +++ & $26-40$ \\
\hline fair & ++ & $11-25$ \\
\hline Poor & + & $1-10$ \\
\hline No & - & 0 \\
\hline
\end{tabular}

\section{Results and discussion}

Effect of different solid media on the mycelial radial growth of Mulberry blight pathogen (A. alternata)

The results of cultural studies revealed that PDA was the best medium for supporting the good radial growth of the isolate
Aa-1, Aa-2, Aa-4 and Aa-7 with the maximum radial growth of $90.00 \mathrm{~mm}$ after ten days of inoculation. Whereas, isolate Aa-2 and Aa-6 yielded maximum growth of $90 \mathrm{~mm}$ in Sabouraud's dextrose agar medium. Isolate Aa-8 and Aa-9 in oat meal agar medium. Maximum growth $(90 \mathrm{~mm})$ in corn meal agar ( Aa-7) and in Richards synthetic agar medium Aa4, Aa-6 and Aa-10 was recorded, which was on par with isolate Aa-1(89.67 mm), Aa-9 (88.93) and Aa-8 (88.58 mm) in Richards synthetic medium followed by Aa-10 in Oat meal agar ( 89. mm), Aa-3 in (88.44) Potato dextrose agar medium. Whereas least growth of isolate Aa-1 was shown on Corn meal agar $(64.46 \mathrm{~mm})$ which was followed by Isolate Aa-2 (76.11), Aa-3 (71.67) in Takahashii's medium (Table 2). The results of the cultural studies on A. alternata causing leaf blight of turmeric showed that among 10 solid media evaluated, radial growth of A. alternata was maximum on potato dextrose and Richards's agar, while maximum sporulation was observed in host leaf extract agar (Gorawar et al. 2006) ${ }^{[1]}$.

Nagrale et al. (2013) ${ }^{[5]}$ observed that the fungus A. alternata causing fungal blight in gerbera, produced profuse mycelium on potato dextrose agar (PDA) with an average width of 4.42 $\mu \mathrm{m}$ in diameter, conidiophores, conidia and intercalary chlamydospores measured as 42.26 x $4.29 \mu \mathrm{m}, 47.16 \times 13.49$ $\mu \mathrm{m}$ and $7.22 \mu \mathrm{m}$ in diameter, respectively. The synthetic media viz., Leonions's agar, Glucose-peptone agar and Sabourand's agar and non-synthetic media, Oat meal agar and PDA were excellent for the mycelial growth and conidial production of $A$. alternata. These results were in agreement with findings of Poornima and Hegde (2014) ${ }^{[6]}$ wherein they opined that PDA and Oat meal agar media were the best media for the growth and sporulation of Cercospora beticola Sacc. These results were in validation with the findings of Poornima (2010) ${ }^{[7]}$ wherein she reported that PDA and Oat meal agar supported the growth of $C$. betiocola.

\section{Effect of different liquid media on the dry mycelial weight of Mulberry blight pathogen ( A. alternata )}

Dry weight of mycelium of the different isolates of mulberry blight pathogen ( A. alternata) was studied in eight different broth media to choose the best broth medium that would support maximum growth. The average dry mycelial weight of the fungus was recorded, ten days after inoculation (Table 3 ). The results of cultural studies revealed that isolate Aa3 showed maximum dry mycelial weight of the fungus as evaluated in Host leaf extract broth $(998.71 \mathrm{mg}$ ) which was followed by Isolate $\mathrm{Aa} 2(997.93 \mathrm{mg}$ ) and Isolate Aa-5 (997.4mg) in Sabouraud's dextrose broth.

Isolate Aa-4 (997.33mg) and Isolate Aa-5 (997.49mg) in oat meal broth and isolate Aa-7 (996.44mg) in Yeast Extract broth. Remaining all other isolates documented dry mycelial weight was on par with each other. Whereas, lowest dry weight of mycelia was in isolate Aa-8 (757.17mg) on Host Leaf extract broth which was followed by isolate Aa-9 (758.30 mg) on Richard's synthetic broth medium

The findings of Reddy et al., 2019 are in line with the various culture media tested that revealed preference of A. alternata to PDB exhibit black to brownish colour colony, maximum growth with excellent sporulation, with large sized conidia (42.15- $47.16 \mu \mathrm{m})$. These results were contradictory with the findings of Shranamma (2012), ${ }^{[11]}$ wherein she reported that highest mean dry mycelial weight was obtained in Richards synthetic broth and none of the media favoured sporulation of Cercoapora musae.

\section{Sporulation of the different isolates of Mulberry blight pathogen $A$. alternata}

To understand good supporting media for sporulation of $A$. alternata a study was carried out on different broth media and 
the results are presented in the table 4. A very good sporulation of the Isolate Aa-1 on Potato dextrose broth, Host leaf extracts broth, Oat meal agar broth and Corn meal broth was recorded. The Potato dextrose broth also supported good sporulation of three isolates viz; Aa-1, Aa-5 and Aa-9.

Host leaf extract broth and corn meal broth supported good sporulation of Isolate Aa-4 and Aa-5 respectively. Whereas, Takahashis broth poorly supported and just sporulation was observed in Isolate Aa-4, Aa- 8 and Aa-10 and remaining all other isolates no sporulation was recorded. Similar trend was observed on Richard's synthetic broth, a good sporulation of isolate Aa-10, Aa-1 and Aa-5 and remaining other isolates with no sporulation on the above said media. Finally on Yeast extract broth all isolates produced no sporulation except isolate Ac-9 and Ac-10 which were poor sporulated. Findings of Ramjegathesh and Ebenezar (2012) ${ }^{[9]}$, revealed that the isolates produced light brown conidia with muriform shape, but the isolates varied in the size (length, width and beak length), abundance of the conidia, the number of cells per conidium and sporulation times.

It was found that mycotoxin production and spore formation of A. alternata are regulated by light in opposite ways. Whereas spore formation was largely decreased under light conditions, the production of $\mathrm{AOH}$ was stimulated 2- to 3-fold (Pruss et al., 2014) ${ }^{[8]}$. Similar results were found with the findings of Reddikumar et al. (2015) [10] wherein they observed varied coloured colonies of $A$. caricae on PDA and Cooks No.2 media with varied pigmentation and excellent sporulation on PDA medium. These results were not in tune with the results of Sharanamma (2012) ${ }^{[11]}$ wherein there was no sporulation of Cercospora musae in PDA medium. Sporulation of $A$. alternata f. sp. sphenocleae using the sporulation medium (S-medium) technique was rapid. Conidia were produced within $24 \mathrm{~h}$ and continuous sporulation was still observed until $120 \mathrm{~h}$. The best primary agar media for conidia production were PDA, 1/2 PDA and VJA, while water agar was the poorest of A. alternata $\mathrm{f}$. sp. sphenocleae conidia (Masangkay et al., 2000) ${ }^{[4]}$.

Table 2: Radial growth of isolates of A. alternata on different solid media

\begin{tabular}{|c|c|c|c|c|c|c|c|c|c|c|c|}
\hline \multirow{3}{*}{ Sl. No. } & \multirow{3}{*}{ Solid media } & \multicolumn{10}{|c|}{ Radial growth (mm) (8-DAI) } \\
\hline & & \multicolumn{10}{|c|}{ ISOLATES } \\
\hline & & Aa-1 & Aa-2 & Aa-3 & Aa-4 & Aa-5 & Aa-6 & Aa-7 & Aa-8 & Aa-9 & Aa-10 \\
\hline 1. & Potato Dextrose Agar & 90.00 & 90.00 & 88.44 & 81.51 & 90.00 & 81.00 & 90.00 & 81.11 & 85.83 & 78.65 \\
\hline 2. & Host Leaf Extract Agar & 82.83 & 81.67 & 84.87 & 87.33 & 84.74 & 76.67 & 87.50 & 85.64 & 80.33 & 86.54 \\
\hline 3. & Sabouraud's Dextrose Agar & 87.62 & 90.00 & 81.33 & 82.77 & 88.31 & 90.00 & 82.11 & 84.48 & 76.19 & 82.54 \\
\hline 4. & Takahashii's Medium & 77.96 & 76.11 & 71.67 & 82.19 & 84.64 & 75.38 & 87.55 & 81.62 & 88.29 & 80.00 \\
\hline 5. & Oat Meal Agar & 84.22 & 87.79 & 82.91 & 88.11 & 85.84 & 85.87 & 85.20 & 90.00 & 90.00 & 89.00 \\
\hline 6. & Corn Meal Agar & 64.46 & 81.13 & 77.31 & 86.14 & 88.43 & 84.74 & 90.00 & 88.28 & 75.86 & 85.50 \\
\hline 7. & Richard's Synthetic Agar & 89.67 & 87.57 & 88.00 & 90.00 & 85.94 & 90.00 & 86.20 & 88.58 & 88.93 & 90.00 \\
\hline 8. & Yeast Extract Agar & 86.09 & 81.89 & 85.51 & 85.80 & 84.64 & 80.34 & 86.80 & 87.69 & 88.50 & 85.56 \\
\hline \multicolumn{2}{|r|}{$\mathbf{F}$} & $* *$ & $* *$ & $* *$ & $* *$ & $* *$ & $* *$ & $* *$ & $* *$ & & \\
\hline \multicolumn{2}{|r|}{ SE m \pm} & 1.45 & 1.57 & 1.62 & 1.61 & 1.44 & 1.6 & 0.91 & 1.11 & 1.19 & 0.93 \\
\hline \multicolumn{2}{|r|}{ CD @ $@=0.01$} & 6.10 & 6.61 & 6.83 & 6.8 & 6.04 & 7.03 & 3.83 & 4.69 & 5.01 & 3.92 \\
\hline
\end{tabular}

** Significant at $1 \%$ level DAI- Days after Incubation

Table 3: Dry mycelial weight of isolates of A. alternata in different liquid media

\begin{tabular}{|c|c|c|c|c|c|c|c|c|c|c|c|}
\hline \multirow{3}{*}{ Sl. No. } & \multirow{3}{*}{ Liquid media } & \multicolumn{10}{|c|}{ Dry mycelial weight (mg) (8-DAI) } \\
\hline & & \multicolumn{10}{|c|}{ ISOLATES } \\
\hline & & $\mathbf{A a} 1$ & $\mathbf{A a} 2$ & $\mathbf{A a}_{3}$ & Aat & Aa5 & Aa6 & Aa7 & Aas & Aa9 & Aa10 \\
\hline 1 & Potato Dextrose broth & 980.61 & 959.02 & 950.58 & 948.00 & 856.00 & 960.00 & 928.21 & 967.10 & 890.11 & 851.22 \\
\hline 2 & Host Leaf Extract broth & 880.44 & 785.52 & 998.71 & 955.43 & 848.00 & 789.00 & 800.19 & 757.17 & 889.08 & 865.18 \\
\hline 3 & Sabouroud's Dextrose broth & 896.50 & 997.93 & 820.77 & 896.03 & 997.41 & 982.18 & 936.11 & 852.07 & 987.18 & 950.41 \\
\hline 4 & Takahashii's broth & 997.28 & 854.50 & 992.33 & 980.07 & 980.00 & 886.00 & 951.08 & 977.15 & 897.81 & 866.11 \\
\hline 5 & Oat Meal broth & 884.41 & 867.44 & 988.27 & 997.33 & 997.49 & 986.33 & 987.03 & 969.03 & 882.12 & 954.22 \\
\hline 6 & Corn Meal broth & 964.43 & 931.17 & 994.33 & 895.17 & 841.83 & 867.74 & 951.13 & 987.87 & 855.18 & 984.26 \\
\hline 7 & Richard's Synthetic broth & 988.33 & 887.04 & 929.67 & 986.25 & 870.10 & 785.18 & 955.10 & 968.27 & 758.30 & 897.07 \\
\hline 8 & Yeast Extract broth & 930.00 & 988.87 & 981.33 & 956.28 & 984.11 & 901.07 & 996.44 & 856.00 & 797.74 & 805.21 \\
\hline & $\mathrm{F}$ & *** & $* *$ & $* *$ & $* *$ & *** & $* *$ & ** & $* *$ & $* *$ & $* *$ \\
\hline & SE $m \pm$ & 1.06 & 0.80 & 1.79 & 0.93 & 1.09 & 0.96 & 0.83 & 0.74 & 0.71 & 0.47 \\
\hline & $C D @ P=0.01$ & 4.45 & 3.37 & 7.57 & 3.92 & 4.57 & 4.02 & 3.52 & 3.12 & 2.97 & 2.0 \\
\hline
\end{tabular}

** Significant at $1 \%$ level; DAI- Days after Incubation

Table 4: Sporulation of isolates of A. alternata on different liquid media

\begin{tabular}{|c|c|c|c|c|c|c|c|c|c|c|c|}
\hline \multirow{3}{*}{ Sl. No. } & \multirow{3}{*}{ Liquid media } & \multicolumn{10}{|c|}{ Sporulation (8 DAI) } \\
\hline & & \multicolumn{10}{|c|}{ ISOLATES } \\
\hline & & AC1 & AC2 & AC3 & AC4 & AC5 & AC6 & AC7 & AC8 & AC9 & $\mathbf{A C 1 0}$ \\
\hline 1 & Potato Dextrose broth & +++ & ++ & + & + & +++ & ++ & + & ++ & +++ & + \\
\hline 2 & Host Leaf Extract broth & +++ & + & + & +++ & + & + & + & + & ++ & + \\
\hline 3 & Sabouroud's Dextrose broth & + & + & ++ & + & $=$ & - & + & + & ++ & + \\
\hline 4 & Takahashii's broth & & & & + & & & & + & - & + \\
\hline 5 & Oat Meal broth & +++ & + & + & + & + & + & + & + & - & - \\
\hline 6 & Corn Meal broth & +++ & + & + & + & +++ & & + & + & + & ++ \\
\hline 7 & Richard's Synthetic broth & + & 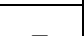 & 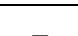 & - & + & 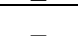 & - & 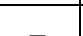 & - & ++ \\
\hline 8 & Yeast Extract broth & - & _- & _ & - & - & _- & - & - & + & + \\
\hline
\end{tabular}

DAI- Days after Incubation

Poor sporulation $(+)(1-10$ conidia); Fair sporulation $(++)(11-25$ conidia); Good sporulation $(+++)$ (26-40 conidia); Very good sporulation (++++) (>40 conidia); No sporulation (-); Number of conidia per microscopic field under 10x considered for categorization 


\section{Conclusion}

The variation was noticed among all the isolates of $A$. alternata in present study with regard to mycelia growth and amount of sporulation on different culture media tested. Majority of the isolates showed better mycelia growth and sporulation in Potato dextrose medium and Oat meal medium with variations. The present findings also deciphered that these two media are better for obtaining rich mycelia growth of A. alternata and could aide in molecular studies. Further, the variation among the isolates needs to be authenticated by virulence and molecular studies.

\section{References}

1. Gorawar MM, Hegde Y, Kulkarni S, Kalappanavar I. Cultural studies on Alternaria alternata (Fr.) Keissler causing leaf blight of turmeric. Int. J. Plant Sci 2006;22:35-38.

2. Gunasekhar V, Govindaiah, Datta RK. A new leaf blight of mulberry (Morus spp.) caused by Alternaria alternata in India. Indian J Seric 1992;31(2):131-134.

3. Leslie JF. Summerell BA. Fusarium Laboratory work shop a recent history. Mycotoxin Research. 2006;22(2):73-74.

4. Misaghi IJ, Grogan RG, Duniway JM, Kimble KA. Influence of environment and culture media on spore morphology of Alternaria alternata. Phytopathol 1978;68:29-34.

5. Nagrale TD, Gaikwad PA, Sharma L. Morphological and cultural characterization of Alternaria alternata (Fr.) Keissler blight of gerbera (Gerbera jamesonii H. Bolus ex J.D. Hook). J Appl. Nat. Sci 2013;5(1):171-178.

6. Poornima, Yashoda R Hegde. Cultural characteristics of Cercospora beticola sacc. causing leaf spot of palak. J Plant Protec 2014;7(2):441-443.

7. Poornima. Studies on cercospora beticola sacc. Causing leaf spot of palak (beta vulgaris var. bengalensis Hort.) M.Sc. (Agri) Thesis, Univ. Agric. Sci., Dharwad (India) 2010.

8. Pruss S, Fetzner R, Seither K, Herr A, Pfeiffer E, Metzler M. Role of the Alternaria alternata blue-light receptor LreA (White Collar 1) in spore formation and secondary metabolism. Appl. Environ. Microbiol 2014;80(8):25822591.

9. Ramjegathesh R, Ebenezar EG. Morphological and Physiological characters of Alternaria alternata causing leaf blight disease of onion. Int. J Plant Pathol 2012;3(2):34-44.

10. Reddi Kumar M, Giridhara Krishna T, Raja Reddy K. Survey and Prevalence of Asperisporium caricae, incitant of black leaf spot of papaya and evaluation of certain new fungicidal. Int. J Res. Stud. Agri. Sci 2015;4:10-14.

11. Sharanamma. Studies on sigatoka leaf spot of banana caused by Cercospora musae. Zimm, M.Sc. (Agri) Thesis, Univ. Agric. Sci., Dharwad (India) 2012.

12. Leslie JF, Summerell BA. Fusarium Laboratory work shop a recent history. Mycotoxin Research 2006;22(2):73-74. 